Xu ML, Jiang H, Jin XG et al. Crowd simulation and its applications: Recent advances. JOURNAL OF COMPUTER SCIENCE AND TECHNOLOGY 29(5): 799-811 Sept. 2014. DOI 10.1007/s11390-014-1469-y

\title{
Crowd Simulation and Its Applications: Recent Advances
}

\author{
Ming-Liang $\mathrm{Xu}^{1}$ (徐明亮), Member, CCF, ACM, IEEE, Hao Jiang ${ }^{2}$ (蒋 浩), Member, CCF, ACM, IEEE \\ Xiao-Gang $\mathrm{Jin}^{3}$ (金小刚), Member, ACM, IEEE \\ and Zhigang Deng ${ }^{4, *}$ (邓志刚), Senior Member, IEEE, Member, ACM \\ ${ }^{1}$ School of Information Engineering, Zhengzhou University, Zhengzhou 450000, China \\ ${ }^{2}$ Beijing Key Lab of Mobile Computing and Pervasive Devices, Institute of Computing Technology \\ Chinese Academy of Sciences, Beijing 100190, China \\ ${ }^{3}$ State Key Lab of CAD\&CG, Zhejiang University, Hangzhou 310058, China \\ ${ }^{4}$ Department of Computer Science, University of Houston, Houston, TX 7r204-3010, U.S.A.
}

E-mail: iexumingliang@zzu.edu.cn; jianghao@ict.ac.cn; jin@cad.zju.edu.cn; zdeng4@uh.edu

Received February 26, 2014; revised July 7, 2014.

\begin{abstract}
This article surveys the state-of-the-art crowd simulation techniques and their selected applications, with its focus on our recent research advances in this rapidly growing research field. We first give a categorized overview on the mainstream methodologies of crowd simulation. Then, we describe our recent research advances on crowd evacuation, pedestrian crowds, crowd formation, traffic simulation, and swarm simulation. Finally, we offer our viewpoints on open crowd simulation research challenges and point out potential future directions in this field.
\end{abstract}

Keywords crowd simulation, emergency evacuation, pedestrian crowd, crowd formation, traffic simulation, swarm simulation

\section{Introduction}

Crowds are complex systems containing collections of individuals, such as human groups, animal herds, insect swarms, and vehicle flows, in the same physical environment. Their exhibited collective behaviors are often different from those that they would act when they are alone. Crowds are ubiquitous phenomena with interesting and uncanny spatial, physical, biological, social, and cultural patterns in nature. Crowd simulation in computer graphics dates back to $1980 \mathrm{~s}^{[1]}$. In recent years, it has attracted significant attention from many research fields, not limited to computer animation and simulation, due to its broad applications in a variety of fields including military simulation ${ }^{[2]}$, architectural $\operatorname{design}^{[3-5]}$, safety science ${ }^{[6-9]}$, entertainment ${ }^{[10-11]}$, physics $^{[12]}$ psychology ${ }^{[13]}$ training systems ${ }^{[14]}$, robotics $^{[15]}$, sociology ${ }^{[16-17]}$, city planning ${ }^{[18]}$, traffic engineering ${ }^{[19-27]}$, insect swarm simulation ${ }^{[28-31]}$, and culture computing ${ }^{[32]}$.
Although there have been numerous research progresses and demonstrated applications in crowd simulation, it is still a rapidly growing area. Indeed, to date, many crowd simulation research challenges still remain widely open due to highly complex behaviors driven by individuals depending on various physiological, psychological and social factors.

To manage the scope of this article, we do not attempt to fully survey crowd simulation techniques that have been proposed during the past several decades. We refer interested readers to recent comprehensive surveys ${ }^{[33-40]}$ for more details. Instead, in this article, after briefly reviewing mainstream crowd simulation methodologies, we will switch its focus to our recent research advances in this research field.

The remainder of this article is organized as follows. We first give a high-level overview on existing crowd simulation methodologies. Then, we present our recent research advances on crowd evacuation, pedestrian crowds, crowd formation, traffic simulation, and

Regular Paper

This work was supported in part by the National Natural Science Foundation of China under Grant Nos. 61202207, 61100086, 61272298, 61210005, 61472370, 61170214, and 61328204, the National Key Technology Research and Development Program of China under Grant Nos. 2013BAH23F01, 2013BAK03B07, and 2013BAK03B0, the Postdoctoral Science Foundation of China under Grant Nos. 2012M520067 and 2013T60706, the National Nonprofit Industry Specific Program of China under Grant No. 2013467058, and the Research Fund for the Doctoral Program of Higher Education of China under Grant No. 20124101120005.

* Corresponding Author

(C) 2014 Springer Science + Business Media, LLC \& Science Press, China 
swarm simulation. Finally, we offer our remarks on open research challenges in this field and point out potential research directions.

\section{Crowd Simulation Methodologies}

Overall, crowd simulations can be divided into two broad areas: focusing on the realism and focusing on the high-quality visualization. In the first case, the visualization of simulations (such as evacuation and training applications) is not crucial, and often a simple yet intuitive 2-dimentional (2D) simulation is sufficient. The visualization is only used to help users to better understand the simulation process. While in the second case (applications such as film production and video games), the emphasis is primarily on high-quality rendering and animation techniques to achieve convincing visual effects.

In this article, we mainly focus on how to model the natural crowd movement - how collective individuals move around and avoid collisions in complex and dynamic environments. To date, many crowd simulation methodologies have been developed to model crowd movement, which can be roughly classified into macroscopic (continuum-based) and microscopic (agent-based) models.

Macroscopic models regard the crowd system as a whole, and are usually designed to achieve real-time simulation for very large crowds, where each individual's behavior is not the primary focus and typically follows the characteristics of the flow as long as the overall crowd movement looks realistic. In contrast, microscopic models focus on individual behaviors and their interactions using complex cognitive models. Although microscopic models are only for smaller crowds to achieve real-time simulation, they can simulate agents in a crowd with more realistic autonomous behaviors.

\subsection{Macroscopic Models}

Macroscopic models use an analogy with fluid or gas dynamics to describe how crowd density and velocity change over time using partial differential equations ${ }^{[12]}$. Based on this, a continuum theory for the flow of pedestrians was proposed by Hughes ${ }^{[41-42]}$ and then many further studies ${ }^{[19,43-44]}$ have extended this method. Macroscopic models can be of value in simulating largescale crowds and highly concentrated populations in spots such as stadiums, shopping malls, and subways.

\subsection{Microscopic Models}

Microscopic models can be traced back to Reynold's seminal work of Boids and steering models ${ }^{[45-46]}$. A large body of further work has accounted for force- based methods ${ }^{[8,47-54]}$, psychological effects ${ }^{[55-60]}$, gridbased methods ${ }^{[61-62]}$, biomechanical models ${ }^{[63-64]}$, sociological factors ${ }^{[65]}$ directional preferences ${ }^{[66]}$, geometrically-based algorithms ${ }^{[67-74]}$, velocity-based models ${ }^{[75-77]}$, field-based models ${ }^{[43,66,75,78-80]}$, cognitive models ${ }^{[81-82]}$, synthetic vision-based models ${ }^{[83]}$, Bayesian decision processes ${ }^{[84]}$, example-based or data-driven methods ${ }^{[25-27,80,85-96]}$, divergence-free flow tiles $^{[97]}$, semantic model ${ }^{[98-99]}$, and many other models of pedestrian behavior ${ }^{[76,96,100-104]}$. Relative to macroscopic models, microscopic models describe every individual's behavior more realistically. However, their main disadvantage is that agents appear to shake or vibrate in high-density crowds.

\section{Our Recent Advances}

During the past decade, we have conducted a variety of crowd simulation researches and have applied them to a number of selected applications. In this article, we roughly divide these research advances into the following categories: crowd evacuation, pedestrian crowds, crowd formation, traffic simulation, and swarm simulation.

\subsection{Crowd Evacuation}

Crowd evacuation is one of the most dominant applications of crowd simulation for safety science and architecture design ${ }^{[105]}$. The goal of such applications is to suggest people how to evacuate crowds safely and efficiently when emergency (such as fires and earthquakes) occurs, and help designers to assess risk and optimize the safe design of architecture space.

\subsubsection{Navigation}

We present a novel method ${ }^{[106-107]}$ for crowd navigation in complex environments based on the continuum model. As illustrated in Fig.1, large and complex environments with multi-constructions can be represented and organized before simulation. Then, the density and discomfort conversion methods are used to keep plausible distance between pedestrians and obstacles when simulating a congested crowd (shown in Fig.2). At last, the comparison experiment shows a high degree of similarity between the real data and the simulation result.

Also, our work ${ }^{[108]}$ presented a semantic model for representing the complex environment, where the semantic information is described with three levels: a geometric level, a semantic level, and an application level.

Each level contains different maps for different purposes, and the interactions between individuals facili- 


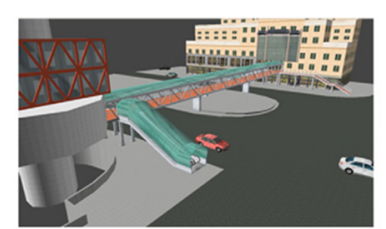

(a)

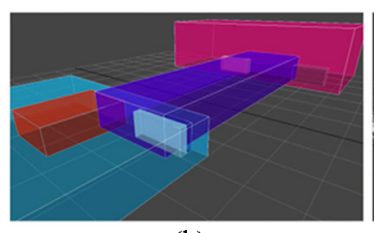

(b)

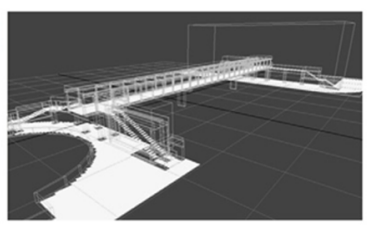

(c)
Fig. ${ }^{[106]}$. (a) 3D geometric models. Sketch map of objects are illustrated.

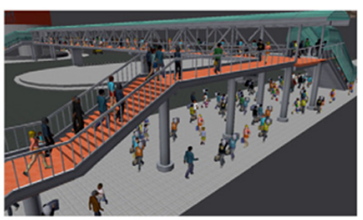

(a)

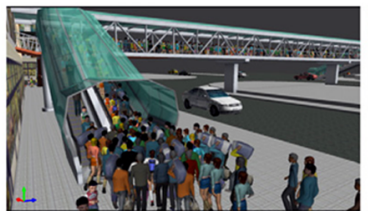

(b)

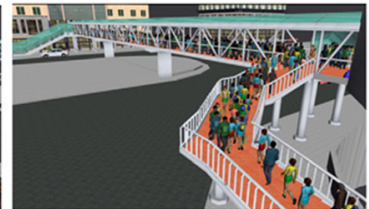

(c)
Fig.2. Selected simulation results ${ }^{[106]}$.

tate the virtual environment. Then, a new method ${ }^{[106]}$ was designed to fit the proposed virtual environment model so that realistic behaviors of large dense crowds could be simulated in multi-layered complex environments such as buildings and subway stations.

\subsubsection{Parallelizing Simulation}

To significantly increase the simulation efficiency, we developed a novel parallelizing approach for crowd simulators constructed with a continuum model rather than an agent-based model ${ }^{[108]}$. Its main idea is to di- vide a crowded virtual environment into some districts connected with transitional blocks, thereby decomposing the simulation into sub-tasks. Then, we designed a two-layered planning method to guide agents within a partitioned large-scale environment, and the details of transiting agents from one district to another have also been presented. We improved the continuum models through parallelization while preserving their existing superiority of generating smooth motion. To this end, our partitioning method effectively simplifies the intricacy of simulation for most large-scale crowd simulation applications.

\subsubsection{Panic Phenomenon}

Special rules or parameter settings are typically needed to simulate a panic phenomenon at a given scheme, thereby we designed a panic model named PPIB $^{[109]}$ (Panic, Propagation and Influence on Behavior), which could evoke panic automatically under dangerous situations without manual intervention. There are three perspectives to describe the panic, including human mental factors and their variations caused by local situation, panic propagation within the crowd, and the influence of panic over the basic factors of pedestrian dynamics.

\subsubsection{Evacuation System}

Simulating crowds in complex environments is fascinating and challenging. Large-scale public places and public facilities are areas with highly concentrated populations. It is necessary to make emergency schemes to safely evacuate people under emergency situations for such areas in advance. With virtual reality techniques, we could visualize the 3D scenes of evacuation and statistically analyze the results. It is instrumental and useful to verify the rationality and validity of a given emergency scheme. Based on our technologies mentioned above, we built a system named Guarder ${ }^{[105]}$, which is a virtual drilling system for crowd evacuation under emergency scheme. Its framework is shown in Fig.3. Its key technologies include semantic description

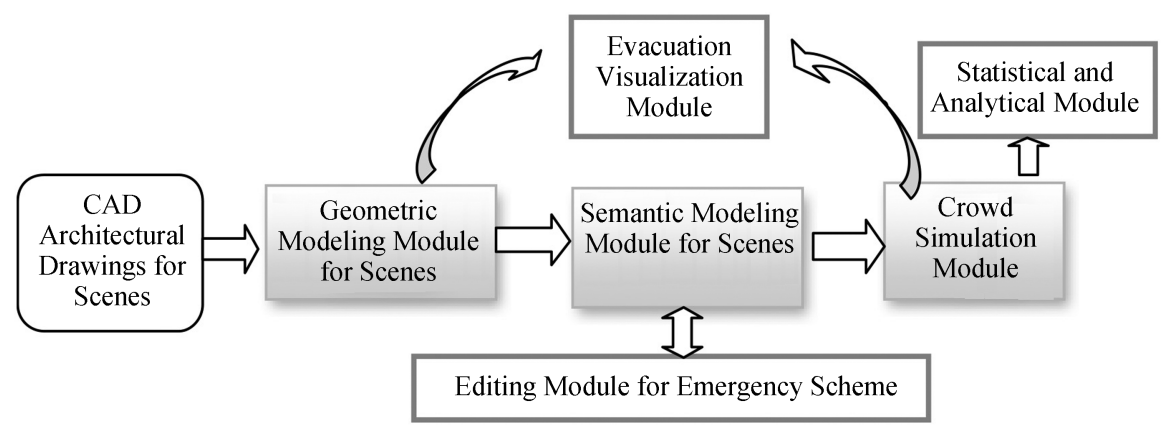

Fig.3. Technical framework of virtual drilling for crowd evacuation. 
for complex environments, crowd simulation, and so on (introduced above).

Finally, it is noteworthy that we have successfully applied our Guarder system to many real-world applications, including the evacuation strategy analysis on the National Stadium known as the Bird's Nest, the pedestrians' guidance on the holiday rush of Beijing West Railway Station, and the pedestrians' analysis on the rush time of the Beijing Olympic Park subway station.

\subsection{Pedestrian Crowds}

Pedestrian crowds frequently appear in many blockbuster feature animation films and video games. Typical examples include pedestrians walking in the street, soldiers fighting in a battle, and spectators watching a performance.

\subsubsection{Simulation Based on Vector Field}

We designed a hybrid architecture solution ${ }^{[110-111]}$ to control the navigation of large-scale agents interactively in a simple but effective way. Our solution models each individual as an agent with his/her own personality, whose behavior is determined by both the global and local movement parts. The global part is derived from a vector field specified by users, which is generated by the anisotropic radial basis functions (RBF) based vector interpolation. The local one contains a velocity-proportional wandering and a weighted movement derived from the positions of anchor points. With this governing tool, our results (shown in Fig.4) demonstrate that users can freely and effectively control the flows of the crowd by sketching velocities on anchor points in the scene.

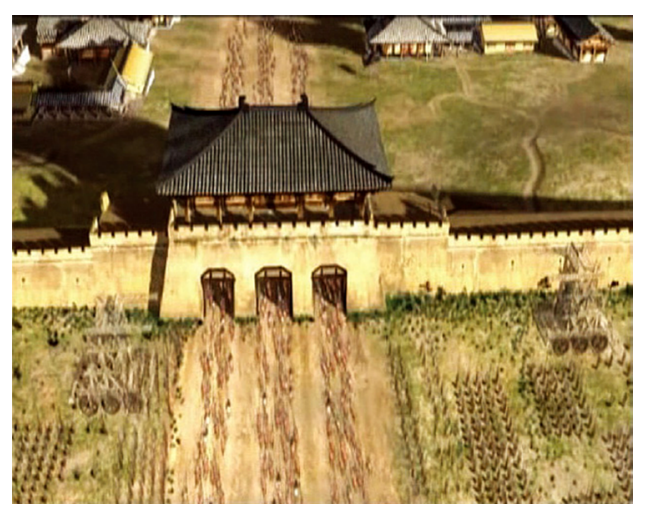

Fig.4. TV screenshot of the medieval city simulation that used our approach ${ }^{[110-111]}$.

\subsubsection{Diversification of Motion Styles}

To efficiently increase a crowd's motion diversity, we developed a novel approach to adaptively control agents' motion styles by maximally reusing a limited number of available motion styles ${ }^{[12]}$. Our method maximizes the style variety of local neighbors and the global style utilization while maintaining a consistent style for each agent as natural as possible. It only requires high-level motion information (such as speed and motion type) computed from the crowd simulation system's navigation and perception layers. As such, it can complement high-level crowd simulation models. Experimental scenarios (shown in Fig.5) and user evaluations (shown in Fig.6) demonstrate our approach's flexibility and capability.

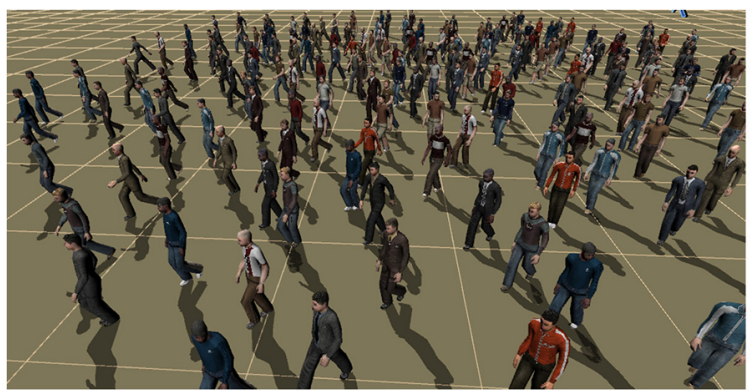

Fig.5. Simulated pedestrian crowds with diversified motion styles $^{[112]}$.

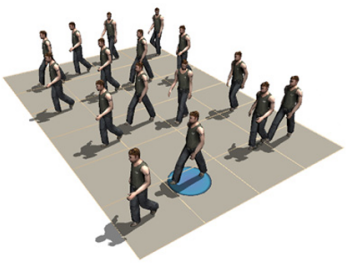

(a)

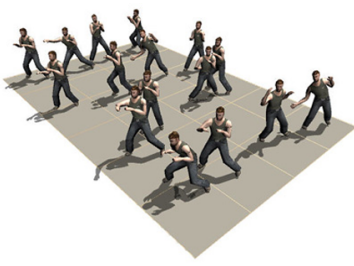

(b)
Fig.6. (a) Cyclic walking motion. (b) Acyclic fighting motion. Our motion diversity control ${ }^{[112]}$ disguises motion clones more effectively than random distribution does, given the same number of available motion styles.

\subsubsection{Perceiving Motion Transitions}

The walking motions of a crowd typically vary in both spatial and temporal domains. However, it is time and memory consuming to create complete motion sequences for every pedestrian. Many collective features such as crowd density, appearance variations, motion variations, and sub-group interaction patterns, affect the perception of motion transitions in a pedestrian crowd.

Therefore, we conducted a series of psychophysical experiments ${ }^{[113]}$ to investigate how these crowd features can influence human perception on walking motion transitions in a crowd when inexpensive motion blending algorithms can be used. Our results (shown in Fig.7) provide practical guidelines for performance- 


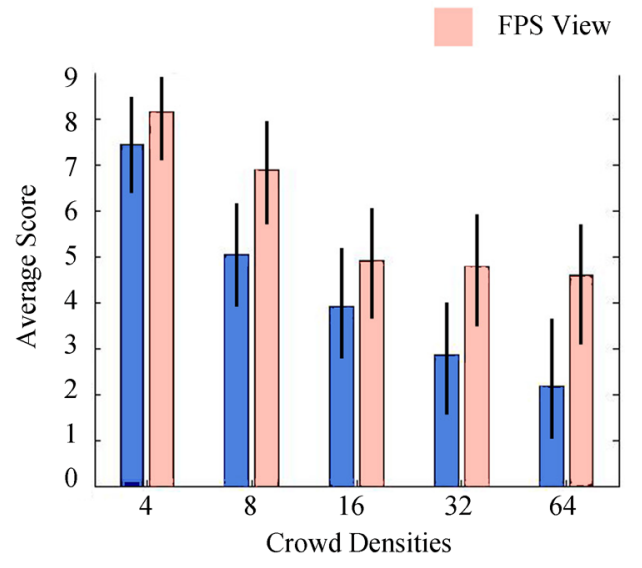

(a)

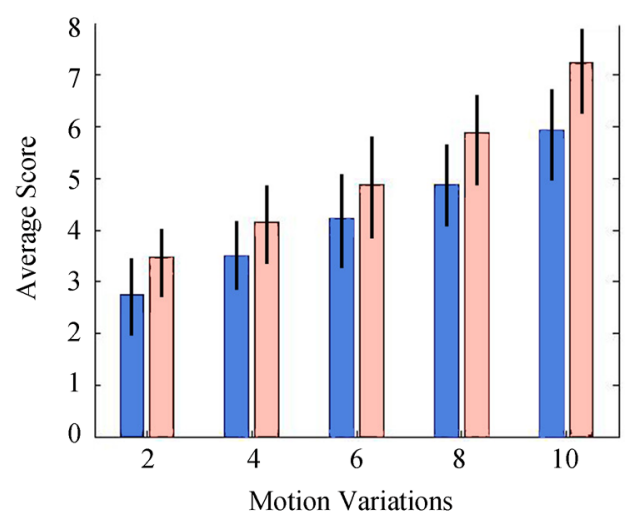

(c)

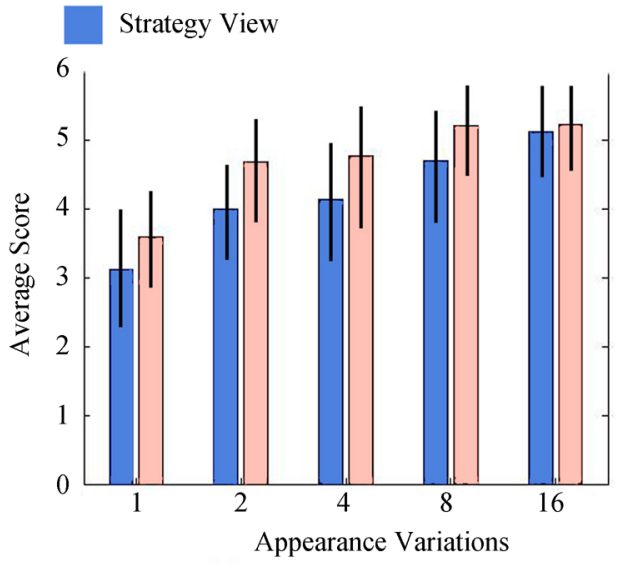

(b)

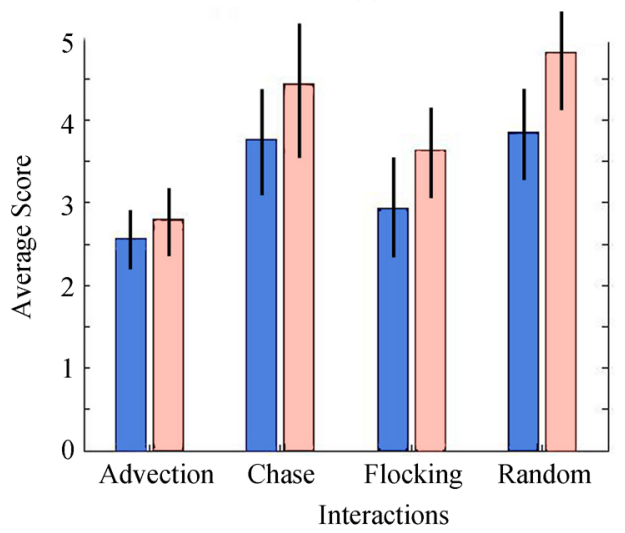

(d)

Fig.7. Evaluation results: average scores and standard deviation errors of different crowd feature experiments ${ }^{[13]}$. FPS: frames per second.

oriented crowd applications such as real-time games to improve the perceptual realism by effectively disguising motion transitions. Specifically, we found that 1) distant viewpoints are more effective in disguising unrealistic pedestrian motion transitions than close viewpoints; 2 ) increasing the density of agent numbers in the viewport can significantly help to hide motion transitions; 3 ) adding more agent appearances (e.g., different textures) does not necessarily lead to better perception of motion transitions in a crowd; and 4) the existence of collective behaviors or sub-group interactions can effectively decrease the negative impact of motion transitions among walking motions.

\subsubsection{Interactive Virtual Marathon}

We developed an exergame called VNM (Virtual Network Marathon) ${ }^{[114]}$, which uses specially devised treadmills for running in an immersive virtual environment. These treadmills with various sensors are designed to collect body performance data. Connected with computers in the Internet, they can control a player's avatar in a virtual world. However, it is dif- ficult for the player to invite many other players to join a network marathon race like in a real-world marathon. Therefore, we employ a number of autonomous virtual players to simulate the marathon race crowd.

In order to generate lifelike competitive running behaviors for virtual players in the crowd (shown in Fig.8), a novel demonstration-based behavior modeling
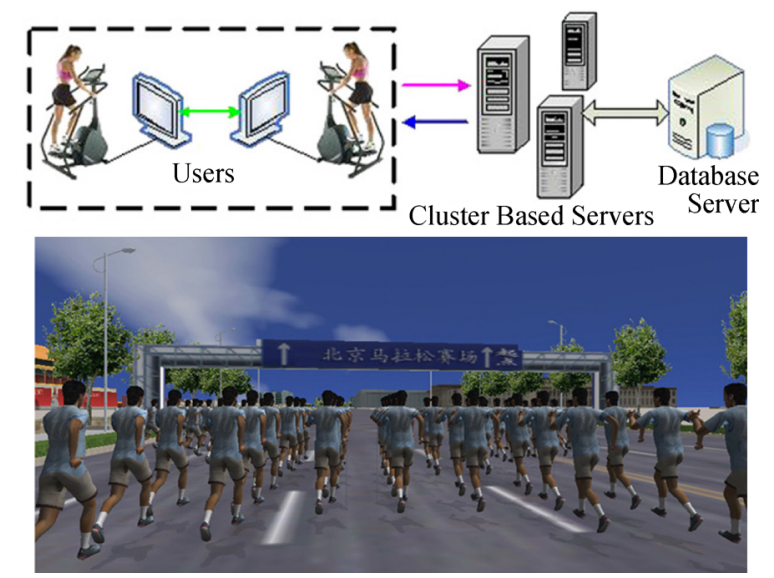

Fig.8. Virtual marathon race crowd ${ }^{[114]}$. 
technique is used. Desired behaviors are learned through mimicking demonstrated running behaviors of real players.

\subsection{Crowd Formation}

Crowd formation has been a relatively new yet important research direction in crowd simulation field, due to its diverse range of applications such as film production, computer games, robotics and performance training.

\subsubsection{Shape-Constrained Flock}

We developed a novel shape-constrained, agentbased flock simulation system ${ }^{[15]}$ that can interactively control flock navigation, and it is capable of making the spatial distribution of a flock to meet user-specified static or deforming shape constraints.

Our approach first draws a set of uniform sample points through a $3 \mathrm{D}$ surface mosaicing process or a stratified point sampling strategy. Then, it establishes correspondences between flock members and sample points on the target shape. Finally, a global path control scheme using the Kalman filter and the fuzzy logic, which dynamically adjusts the force, is employed to create desired flocking animations. Our results (shown in Fig.9) suggest that the generated animation effects are visually desirable and pleasing.
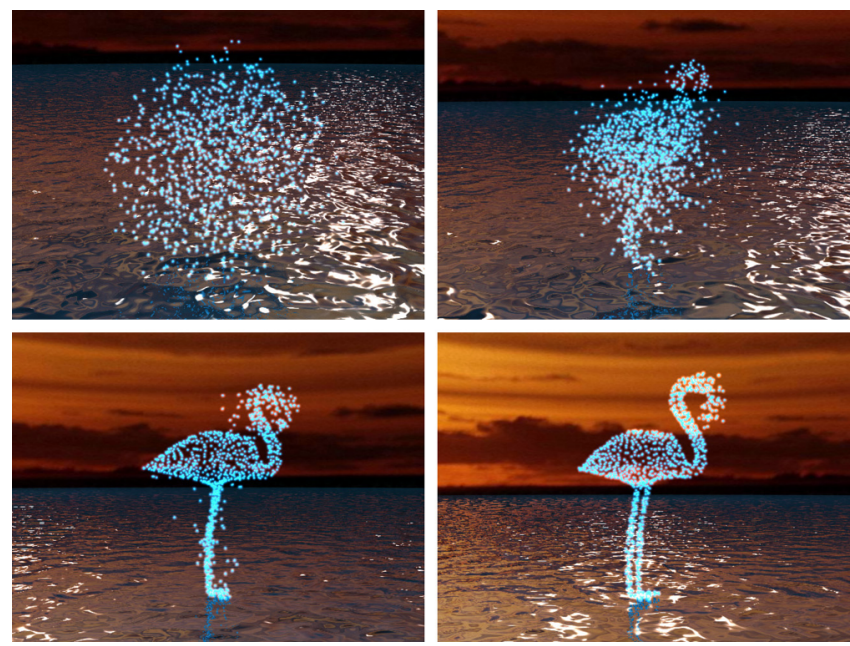

Fig.9. A flock dynamically transforms into a waterfowl shape $^{[115]}$.

\subsubsection{Sketch-Based Formation Control}

We developed an interactive, scalable framework ${ }^{[113]}$ that generates freestyle group formations and transitions by computing a plausible agent distribution in the target formation and agent correspondences between keyframes. Moreover, our approach also provides a two- level formation trajectory control technique that helps users intuitively guide agents' transition paths. Our results (shown in Fig.10) demonstrate this approach's flexibility and effectiveness.

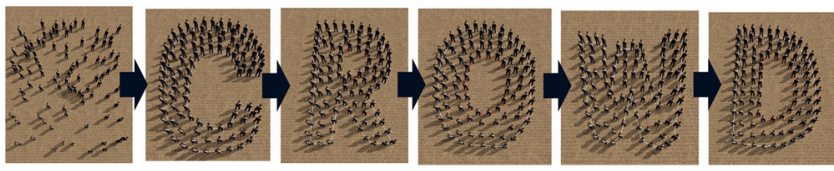

Fig.10. Freestyle group formations ${ }^{[113]}$.

Moreover, we also proposed a solution called "formation sketching" to provide a flexible sketch-based framework to generate arbitrary group formations. This solution, unlike example-based methods limited by the number of training examples, can freely create various precise formations specified by users. Our results (shown in Fig.11) demonstrate that this solution is efficient and adaptive to variations of group scales, group positions and environment obstacles.
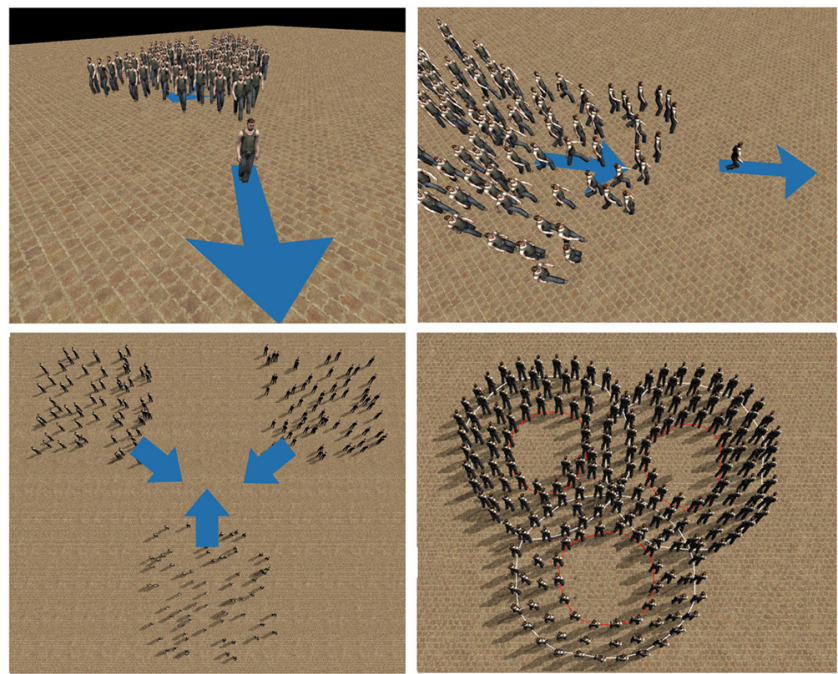

Fig.11. Stylizing groups by formation sketching.

\subsubsection{Collective Formation Transform}

We also developed a novel group formation transform system ${ }^{[116-117]}$ to automatically generate an aesthetically formation transformation while effectively maintaining the stability of local structure and preserving the dynamic collective behavior, given source and target formation shapes.

First, the source and target formation shapes are converted to representations of the Delaunay triangulation (DT), where each vertex represents the spatial position of an agent in its group. Then, the effort of each agent during the transformation is quantified by a novel formula, and a new relative distance variance measure is used to cluster collective subgroups. In 
the transformation, shape manipulation and the Social Force Model (SFM) are extended to ensure collisionfree movements. Finally, the effectiveness and the robustness of our approach are evaluated via quantitative measures, including the mutual information of crowd dynamics, the stability of local structure, and effort balancing. Experimental results (shown in Fig.12) demonstrate that our approach can produce more aesthetically satisfactory and fluid crowd formation transformations than state-of-the-art methods.

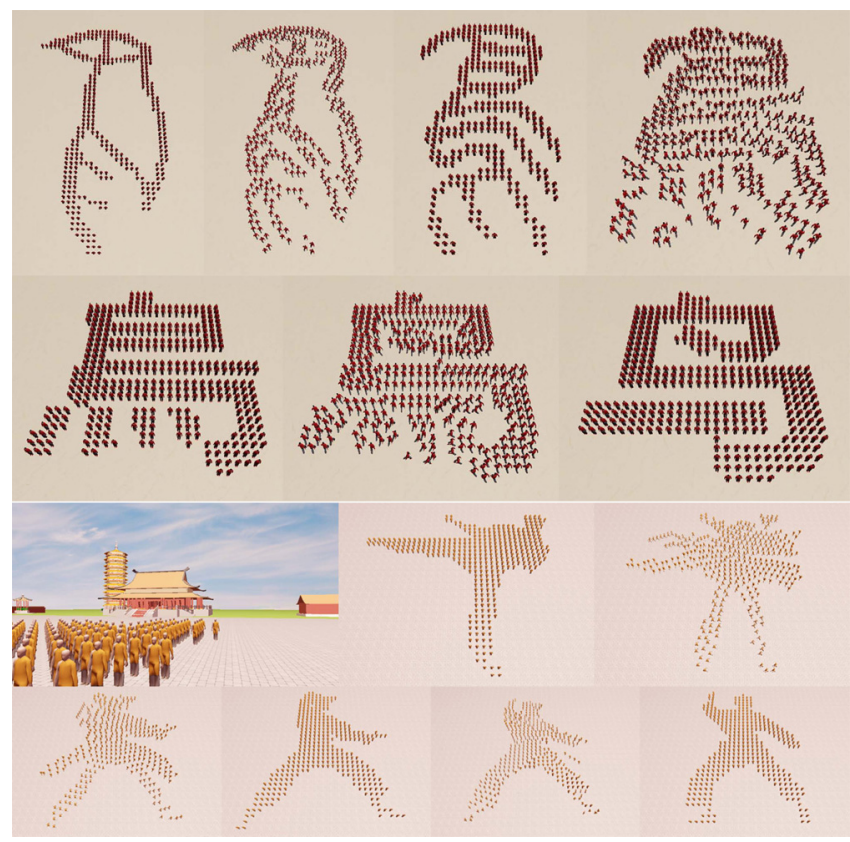

Fig.12. Evolution of the Chinese word "鸟" meaning "bird" in English and the martial arts for 400 agents $^{[116-117]}$.

\subsection{Traffic Simulation}

Traffic simulation, to realistically portray the contemporary world, plays an increasingly important role in virtual worlds, because there is a growing need of that in many fields such as video game, movie, and virtual globe.

\subsubsection{Detailed Urban Road Networks}

We developed an agent-based system ${ }^{[24]}$ to generate detailed traffic animation on urban arterial networks with signalized crossing, merging and weaving areas, and to produce an immersive traffic flow animation with realistic driving and lane-changing behaviors. The popular follow-the-leader method is employed to simulate intelligent driving styles and various vehicle types. Meanwhile, the continuous lane-changing model is used to imitate the driver's decision-making process and the vehicle's dynamic interactions with neighboring vehicles. Results (shown in Fig.13) demonstrate the capa- bility of our approach to visualize complex urban traffics with a rich variety of vehicle types, driving styles, and road topologies.

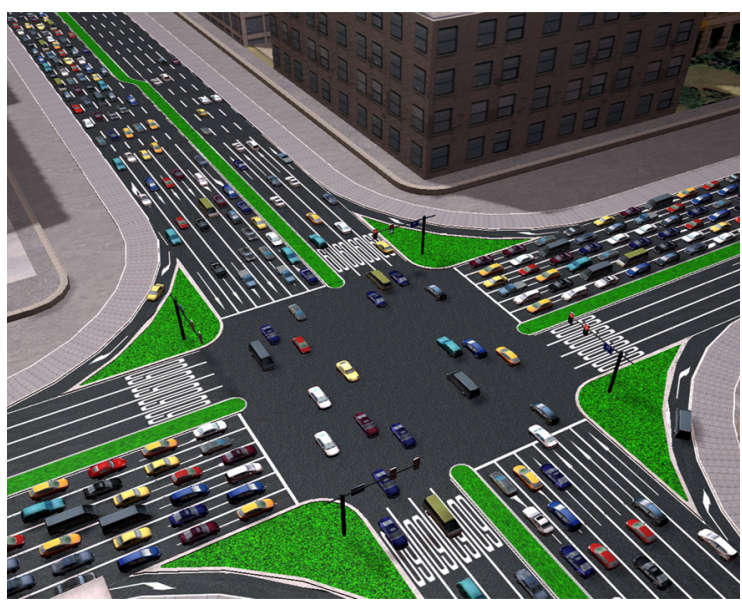

Fig.13. Signalized crossing traffic scenario ${ }^{[24]}$.

\subsubsection{Video-Driven Traffic Simulation}

Existing traffic simulators set drivers' characterized driving parameters, such as speed choice, gap acceptance, preferred rate of acceleration or deceleration, environmental adaptation factor, as random values around the average of empirical values, which cannot simulate the drivers' personalized driving behaviors. Therefore, we developed a data-driven system ${ }^{[23]}$ to simulate virtual traffic flows that exhibit realistic driving behaviors.

Our system learns drivers' specific driving characteristics from video samples. Its main process can be divided into three phases: the acquisition of each vehicle's trajectory data, the learning of each vehicle's unique driving habits, and the online traffic simulation. As a result, given each vehicle's initial status and the personalized parameters as input, our approach can vividly reproduce the traffic flow in the sample video with a high accuracy (shown in Fig.14).
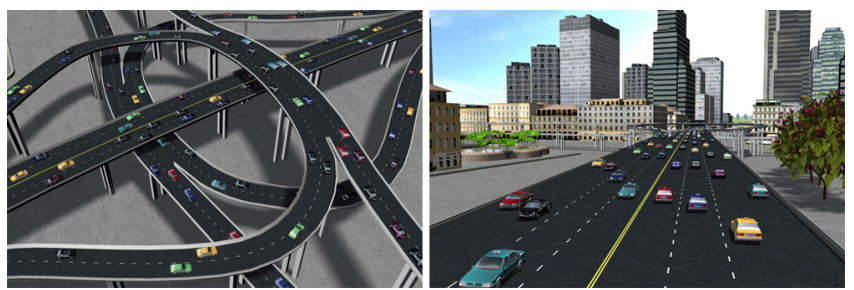

Fig.14. Some simulated traffic scenarios ${ }^{[23]}$

\subsubsection{Predictive Adaptive-AR Model}

The conventional continuum-based models, without taking drivers' behavior into account, usually assume 
vehicles follow the flows passively. To address this issue, we proposed a novel adaptive-AR model ${ }^{[22]}$, based on the continuum-based $\mathrm{AR}$ mode ${ }^{[118]}$, to simulate real-life vehicle traffic flows. Our method extends the AR model with a few factors including the reaction time, the prediction effectiveness, and the types of the drivers.

Our experimental results (shown in Fig.15) show that the adaptive-AR model makes the simulated traffic flows more realistic in real time and better exhibit the drivers' responsive behaviors than the AR model.
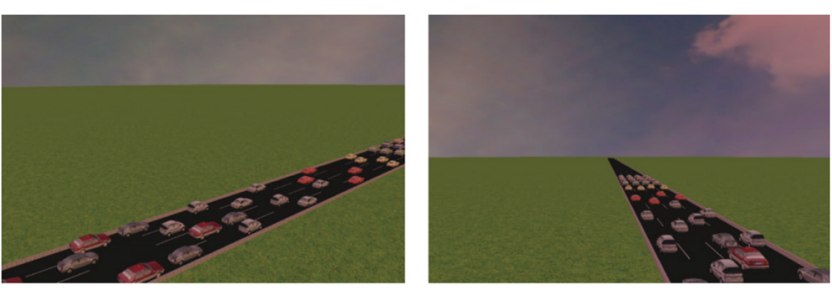

Fig.15. Some simulated traffic scenarios ${ }^{[22]}$

\subsubsection{Animating Realistic Rural Traffics}

We developed a novel agent-based approach called Accident-Avoidance Full Velocity Difference Model $(\mathrm{AA}-\mathrm{FVDM})^{[21]}$ to simulate realistic street-level rural traffics. We also designed a novel scheme to animate the lane-changing maneuvering process. Furthermore, we validated our method using real-world traffic data.

Our experimental results demonstrate, besides addressing the problem of the close-car-braking circumstance (shown in Fig.16), our AA-FVDM method can efficiently (in real time) simulate large-scale traffic flows (tens of thousands of vehicles) with realistic and smooth effects.

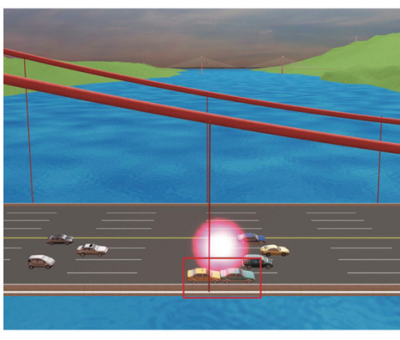

(a)

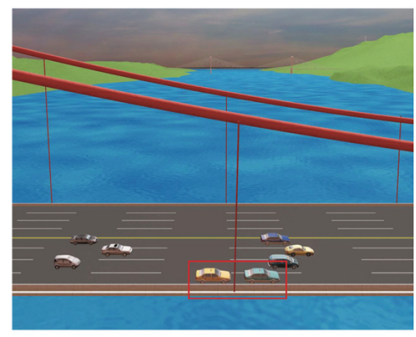

(b)
Fig.16. Simulated scenarios using the existing FVDM method and our method (AA-FVDM) under the close-car-braking circumstance with the same initial conditions ${ }^{[21]}$. (a) Using FVDM. (b) Using our AA-FVDM.

\subsection{Insect Swarm Simulation}

Not limited to humans, various biological insects can often demonstrate a variety of crowd or swarm behaviours in our world. Recently we developed a field- based approach ${ }^{[30]}$ to realistically simulate behaviour patterns of insect swarms. We formulate this simulation problem as a mesoscopic multi-agent system that models both macroscopic global path planning and local behaviours. Its core idea is to construct a smooth yet noise-aware governing velocity field, including two subfields: a divergence-free curl noise field and an enhanced global velocity field. The first sub-field models individual insects' noise-induced movements, while the other controls global navigational paths in a complex environment for the whole swarm. This approach is the first multi-agent modelling system that introduces curl-noise into agents' velocity field and uses its non-scattering nature of the resulting field to maintain noise-aware yet non-colliding movements in $3 \mathrm{D}$ crowd simulation.

Our experimental results (shown in Fig.17) demonstrate that our approach can effectively simulate insect swarms' various realistic behaviours such as massmigrating, positive phototaxis, aggregation, and sedation.

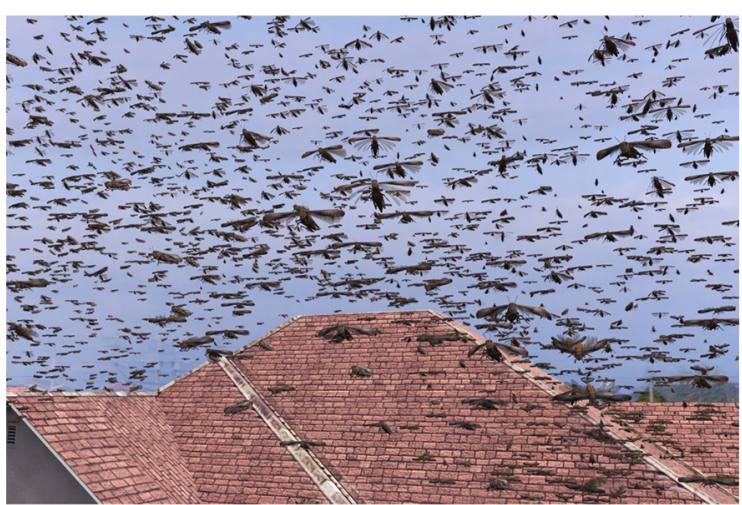

(a)

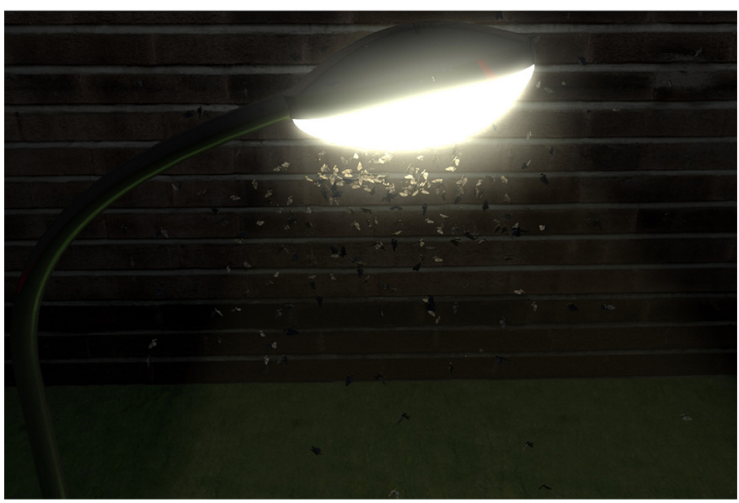

(b)

Fig. $17^{[30]}$. (a) A large swarm of migratory locusts invades a hamlet. (b) A swarm of moths flies around a street lamp.

\section{Challenges and Viewpoints}

Although researchers have extensively studied crowd simulation (algorithms, models, and applications) to date, it is still a relatively young research area. Many 
open research problems are still very much in flux due to the complexity, variety, and dynamics of real-world crowd behaviors.

To date, most of existing crowd simulation methods are essentially application-specific. Indeed, the design of a general, robust, and practical crowd simulation framework that can handle a large variety of applications is still considered as a grand challenge in crowd simulation field. Moreover, many research problems are still widely open and need to be further investigated to advance the applications and practices of crowd simulation. As the final remark, we offer our own viewpoints on these remaining research challenges that are either inadequately studied or largely ignored in the current literature.

1) Modeling the Intelligent Behavior of Rescue Workers or the Authority. Rescue workers or the authority typically play a critical role in the case of emergency evacuation. Different from existing crowd simulation, realistically simulating the behavior of the involved rescue workers or the authority would face different types of technical challenges. For example, the simulation focus would be to model how to convey dynamic emergency information (e.g., safe exits) among evacuees and how to model the dynamics and interaction between the rescue workers and evacuees on the spot. If we can successfully model and incorporate their interactions and dynamic behaviors into emergency evacuation simulation, new simulation results are anticipated to provide much more valuable guidelines and references for the design of more efficient and safer emergency evacuation plans.

2) Modeling and Simulation of Panic Crowds. Panic is a protective psychological reaction when we face sudden and irresistible disasters. It is an unusual action that we often will take when we face unpredictable disasters to save our lives. Although the panic has the characteristics of irrationality and unsociability, it often exhibits specific panic patterns such as herding and so on. Quantitatively understanding the panic pattern and how it is contaminated among people would be of significant importance from the public safety perspective. In our views, the main challenge of simulating panic crowds is the difficulty of acquiring sufficient realworld panic crowd data for crowd model learning and validation.

3) Modeling Confrontational Crowd Behaviors. There are many kinds of confrontational crowd events in the real world such as riots and demonstration. Taking the coupling and derivatives between those events and the crowd evacuation problems into account, it is necessary to combine various established insight and knowledge from the psychology and sociology fields when we want to model the confrontational crowd behavior. Such inputs would be tremendously useful if researchers want to realistically simulate various phenomena using computer algorithms.

4) Automated Model Calibration Based on Instance Data. Since a large number of parameters or factors exist in a crowd simulation system, quantitatively validating the accuracy of a simulation model and automatically calibrating the model's parameters, in order to produce a more realistic behavior of the virtual crowd, is one of widely open research challenges. Instance data is often referred to as the crowd movement datasets acquired from various real-world crowds such as the video of real-world crowds. It would significantly help to produce more credible and realistic crowd simulation results if we can automatically calibrate the parameters of various pedestrian or crowd models.

5) Modeling Extreme Cases Such as the Squeezing and Trampling in the Crowd. The essence of squeezing and trampling accidents in a crowd is the result of accumulating the concurrent and nonlinear contact forces. Building a computational model to describe the condition and evolution of such extreme cases will be of paramount significance to the general public safety. In order to simulate the crowd's realistic responsive behaviours in these extreme cases, including pushing, shoving, crushing, tumbling, trampling, piling up, and so forth, such models should comprehensively reflect real humans' physiological, mental, physical, emotional and appearing characteristics as well as mechanisms. Similar to the panic crowd simulation described previously, the difficulty of acquiring reliable and sufficient real-world crowd behaviour datasets for such extreme cases makes plausible simulation much more challenging than what it may appear.

6) Quantitative, Accurate, and Robust Validation of Various Simulated Crowds. Despite of those exciting research progresses made in the crowd simulation field to date, relatively few efforts have been taken to accurately and automatically validate the realism and plausibility of various simulated crowds ${ }^{[79]}$. Indeed, realism evaluation is often the first problem encountered when researchers or practitioners try to apply any crowd simulation methods to application domains. The design of quantitative, accurate, and robust validation methods for crowd simulation is still considered as a widely open problem. Although certain progresses ${ }^{[93]}$ have been made in this direction very recently, this data-driven method is still limited to specific given training examples.

\section{References}

[1] Thalmann D, Musse S. Crowd Simulation (1st edition). Springer-Verlag, 2007. 
[2] Hawe G I, Coates G, Wilson D T et al. Agent-based simulation for large-scale emergency response: A survey of usage and implementation. ACM Computing Surveys (CSUR), 2012, 45(1): Article No.8.

[3] Schelhorn T, O'Sullivan D, Haklay M et al. STREETS: An agent-based pedestrian model. In Proc. the 6th International Conference on Computers in Urban Planning and Urban Management, April 1999.

[4] Penn A, Turner A. Space syntax based agent simulation. In Proc. the 1st International Conference on Pedestrian and Evacuation Dynamics, 2001, pp.99-114.

[5] Turner A, Penn A. Encoding natural movement as an agentbased system: An investigation into human pedestrian behaviour in the built environment. Environment and Planning B: Planning and Design, 2002, 29(4): 473-490.

[6] Thompson P A, Marchant E W. A computer model for the evacuation of large building populations. Fire Safety Journal, 1995, 24(2): 131-148.

[7] He G Q, Yang Y, Chen Z H et al. A review of behavior mechanisms and crowd evacuation animation in emergency exercises. Journal of Zhejiang University SCIENCE C, 2013, 14(7): 477-485.

[8] Helbing D, Farkas I, Vicsek T. Simulating dynamical features of escape panic. Nature, 2000, 407: 487-490.

[9] Bohannon J. Directing the herd: Crowds and the science of evacuation. Science, 2005, 310(5746): 219-221.

[10] Henry J, Shum H, Komura T. Interactive formation control in complex environments. IEEE Transactions on Visualization and Computer Graphics, 2014, 20(2): 211-222.

[11] Wang X, Zhou L, Deng Z et al. Flock morphing animation. Computer Animation and Virtual Worlds, 2014, 25(3/4): 353-362.

[12] Henderson L. The statistics of crowd fluids. Nature, 1971, 229(5284): 381-383.

[13] Moussaïd M, Helbing D, Theraulaz G. How simple rules determine pedestrian behavior and crowd disasters. Proceedings of the National Academy of Sciences, 2011, 108(17): 6884-6888.

[14] Pelechano N, Badler N I. Modeling crowd and trained leader behavior during building evacuation. IEEE Computer Graphics and Applications, 2006, 26(6): 80-86.

[15] Molnár P, Starke J. Control of distributed autonomous robotic systems using principles of pattern formation in nature and pedestrian behavior. IEEE Transactions on Systems, Man, and Cybernetics, Part B: Cybernetics, 2001, 31(3): 433-435.

[16] McPhail C, Powers W T, Tucker C W. Simulating individual and collective action in temporary gatherings. Social Science Computer Review, 1992, 10(1): 1-28.

[17] Tucker C W, Schweingruber D, McPhail C. Simulating arcs and rings in gatherings. International Journal of HumanComputer Studies, 1999, 50(6): 581-588.

[18] Lin M C, Manocha D. Virtual cityscapes: Recent advances in crowd modeling and traffic simulation. Frontiers of Computer Science in China, 2010, 4(3): 405-416.

[19] Sewall J, Wilkie D, Merrell P et al. Continuum traffic simulation. Computer Graphics Forum, 2010, 29(2): 439-448.

[20] Lin M, Guy S, Narain R et al. Interactive modeling, simulation and control of large-scale crowds and traffic. In Proc. the 2nd International Workshop Motio in Games (MIG), Nov. 2009, pp.94-103.

[21] Lu X, Chen W, Xu M et al. AA-FVDM: An accidentavoidance full velocity difference model for animating realistic street-level traffic in rural scenes. Computer Animation and Virtual Worlds, 2014, 25(1): 83-97.

[22] Lu X, Xu M, Chen W et al. Adaptive-AR model with drivers' prediction for traffic simulation. International Journal of Computer Games Technology, 2013, 2013: Article ID 904154.
[23] Chao Q, Shen J, Jin X. Video-based personalized traffic learning. Graphical Models, 2013, 75(6): 305-317.

[24] Shen J, Jin X. Detailed traffic animation for urban road networks. Graphical Models, 2012, 74(5): 265-282.

[25] Wilkie D, Sewall J, Lin M C. Transforming GIS data into functional road models for large-scale traffic simulation. IEEE Transactions on Visualization and Computer Graphics, 2012, 18(6): 890-901

[26] Sewall J, van den Berg J P, Lin M C et al. Virtualized traffic: Reconstructing traffic flows from discrete spatiotemporal data. IEEE Transactions on Visualization and Computer Graphics, 2011, 17(1): 26-37.

[27] Sewall J, Wilkie D, Lin M C. Interactive hybrid simulation of large-scale traffic. ACM Trans. Graph., 2011, 30(6): Article No.135.

[28] Wu J C, Popović Z. Realistic modeling of bird flight animations. ACM Transactions on Graphics (TOG), 2003, 22(3): 888-895.

[29] Klotsman M, Tal A. Animation of flocks flying in line formations. Artificial Life, 2012, 18(1): 91-105.

[30] Wang X, Jin X, Deng Z et al. Inherent noise-aware insect swarm simulation. Computer Graphics Forum, 2014. (to be appeared)

[31] Tu X, Terzopoulos D. Artificial fishes: Physics, locomotion, perception, behavior. In Proc. the 21st ACM SIGGRAPH, July 1994, pp.43-50.

[32] Anderson E F, McLoughlin L, Liarokapis F et al. Developing serious games for cultural heritage: A state-of-the-art review. Virtual Reality, 2010, 14(4): 255-275.

[33] Schreckenberg M, Sharma S D. Pedestrian and Evacuation Dynamics. Springer-Verlag, 2002.

[34] Thalmann D. Populating virtual environments with crowds. In Proc. the 2006 ACM International Conference on Virtual Reality Continuum and Its Applications, June 2006, p.11.

[35] Pelechano N, Allbeck J, Badler N I. Virtual Crowds: Methods, Simulation, and Control. Morgan and Claypool Publishers.

[36] Pettré J, Kallmann M, Lin M C. Motion planning and autonomy for virtual humans class notes. In Proc. ACM SIGGRAPH 2008 Classes, Aug. 2008, Article No.42.

[37] Shopf J, Barczak J, Oat C et al. March of the Froblins: Simulation and rendering massive crowds of intelligent and detailed creatures on GPU. In Proc. ACM SIGGRAPH 2008 Games, Aug. 2008, pp.52-101.

[38] Huerre S, Lee J, Lin M et al. Simulating believable crowd and group behaviors. In Proc. ACM SIGGRAPH Asia 2010 Courses, Dec. 2010, Article No.13.

[39] Zhou S, Chen D, Cai W et al. Crowd modeling and simulation technologies. ACM Transactions on Modeling and Computer Simulation (TOMACS), 2010, 20(4): Article No.20.

[40] Kapadia M, Badler N I. Navigation and steering for autonomous virtual humans. Wiley Interdisciplinary Reviews: Cognitive Science, 2013, 4(3): 263-272.

[41] Hughes R L. A continuum theory for the flow of pedestrians. Transportation Research Part B: Methodological, 2002, 36(6): 507-535.

[42] Hughes R L. The flow of human crowds. Annual Review of Fluid Mechanics, 2003, 35: 169-182.

[43] Treuille A, Cooper S, Popovic Z. Continuum crowds. In Proc. ACM SIGGRAPH 2006, July 2006, pp.1160-1168.

[44] Narain R, Golas A, Curtis S et al. Aggregate dynamics for dense crowd simulation. ACM Trans. Graph., 2009, 28(5): Article No. 122.

[45] Reynolds C W. Flocks, herds and schools: A distributed behavioral model. In Proc. the 14th ACM SIGGRAPH, July 1987, pp.25-34.

[46] Reynolds C W. Steering behaviors for autonomous characters. In Proc. Game Developers Conference, Mar. 1999. 
[47] Helbing D, Molnar P. Social force model for pedestrian dynamics. Physical Review E, 1995, 51(5): 4282-4286.

[48] Cordeiro O C, Braun A, Silveria C B et al. Concurrency on social forces simulation model. In Proc. the 1st International Workshop on Crowd Simulation, Nov. 2005.

[49] Gayle R, Moss W, Lin M C et al. Multi-robot coordination using generalized social potential fields. In Proc. IEEE International Conference on Robotics and Automation, May 2009, pp.106-113.

[50] Pelechano N, Allbeck J M, Badler N I. Controlling individual agents in high-density crowd simulation. In Proc. ACM SIGGRAPH/Eurographics Symposium on Computer Animation, Aug. 2007, pp.99-108.

[51] Heïgeas L, Luciani A, Thollot J et al. A physically-based particle model of emergent crowd behaviors. arXiv Preprint arXiv:1005.4405, 2010.

[52] Lakoba T I, Kaup D J, Finkelstein N M. Modifications of the Helbing-Molnar-Farkas-Vicsek social force model for pedestrian evolution. Simulation, 2005, 81(5): 339-352.

[53] Sugiyama Y, Nakayama A, Hasebe K. 2-dimensional optimal velocity models for granular flows. In Pedestrian and Evacuation Dynamics, Schreckenberg M, Sharma SD (eds.), 2001, pp.155-160.

[54] Sud A, Gayle R, Anderson E et al. Real-time navigation of independent agents using adaptive roadmaps. In Proc. the 2007 ACM Symposium on Virtual Reality Software and Technology, Oct. 2007, pp.99-106.

[55] Guy S J, Kim S, Lin M C et al. Simulating heterogeneous crowd behaviors using personality trait theory. In Proc. the 2011 ACM SIGGRAPH/Eurographics Symposium on Computer Animation, Aug. 2011, pp.43-52.

[56] Durupïnar F, Pelechano N, Allbeck J M et al. How the ocean personality model affects the perception of crowds. IEEE Comput. Graph. App., 2011, 31(3): 22-31.

[57] Pelechano N, O'brien K, Badler N et al. Crowd simulation incorporating agent psychological models, roles and communication. In Proc. the 1st International Workshop on Crowd Simulation, Nov. 2005, pp.21-30.

[58] Durupïnar F. From audiences to mobs: Crowd simulation with psychological factors [Ph.D. Thesis]. Institute of Engineering and Science, Bilkent University, 2010.

[59] Beltaief O, Hadouaj S, Ghedira K. Multi-agent simulation model of pedestrians crowd based on psychological theories. In Proc. the 4th International Conference on Logistics (LOGISTIQUA), May 31-June 3, 2011, pp. 150-156.

[60] Kim S, Guy S J, Manocha D et al. Interactive simulation of dynamic crowd behaviors using general adaptation syndrome theory. In Proc. ACM SIGGRAPH Symposium on Interactive 3D Graphics and Games, March 2012, pp.55-62.

[61] Loscos C, Marchal D, Meyer A. Intuitive crowd behaviour in dense urban environments using local laws. In Proc. Theory of Practice of Computer Graphics 2003, June 2003, pp.122129.

[62] Alexa M, Muller W. Representing animations by principal components. Computer Graphics Forum, 2000, 19(3): 411418.

[63] Guy S J, Chhugani J, Curtis S et al. PLEdestrians: A leasteffort approach to crowd simulation. In Proc. the 2010 ACM SIGGRAPH/Eurographics Symposium on Computer Animation, July 2010, pp.119-128.

[64] Guy S J, Curtis S, Lin M C et al. Least-effort trajectories lead to emergent crowd behaviors. Physical review E, 2012, 85(1): 016110.

[65] Musse S R, Thalmann D. A model of human crowd behavior: Group inter-relationship and collision detection analysis. In Proc. Eurographics Workshop on Computer Animation and Simulation, Sept. 1997, pp.39-51.
[66] Sung M, Gleicher M, Chenney S. Scalable behaviors for crowd simulation. Computer Graphics Forum, 2004, 23(3): 519-528.

[67] Fiorini P, Shiller Z. Motion planning in dynamic environments using velocity obstacles. The International Journal of Robotics Research, 1998, 17(7): 760-772.

[68] Sud A, Anderson E, Curtis S et al. Real-time path planning in dynamic virtual environments using multiagent navigation graphs. IEEE Transactions on Visualization and Computer Graphics, 2008, 14(3): 526-538.

[69] van den Berg J, Patil S, Sewall J et al. Interactive navigation of multiple agents in crowded environments. In Proc. ACM SIGGRAPH Symposium on Interactive 3D Graphics and Games (I3D), Feb. 2008, pp.139-147.

[70] Guy S J, Chhugani J, Kim C et al. ClearPath: Highly parallel collision avoidance for multi-agent simulation. In Proc. $A C M$ SIGGRAPH/Eurographics Symposium on Computer Animation (SCA), Aug. 2009, pp.177-187.

[71] van den Berg J, Lin M, Manocha D et al. Centralized path planning for multiple robots: Optimal decoupling into sequential plans. Robotics: Science and Systems (RSS), 2009.

[72] Zheng L, Zhao J, Cheng Y et al. Geometry-constrained crowd formation animation. Computers \& Graphics, 2014, 38: 268276 .

[73] Golas A, Narain R, Lin M. Hybrid long-range collision avoidance for crowd simulation. In Proc. the ACM SIGGRAPH Symposium on Interactive $3 D$ Graphics and Games, March 2013, pp.29-36

[74] Lv L, Mao T, Liu X et al. Optimization-based group performance deducing. Computer Animation and Virtual Worlds, 2014, 25(2): 171-184

[75] Patil S, van den Berg J, Curtis S et al. Directing crowd simulations using navigation fields. IEEE Transactions on Visualization and Computer Graphics, 2011, 17(2): 244-254.

[76] Pettré J, Ondrej J, Olivier A H et al. Experiment-based modeling, simulation and validation of interactions between virtual walkers. In Proc. ACM SIGGRAPH/Eurographics Symposium on Computer Animation, Aug. 2009, pp.189-198.

[77] Kim S, Guy S J, Manocha D. Velocity-based modeling of physical interactions in multi-agent simulations. In Proc. the 12th ACM SIGGRAPH/Eurographics Symposium on Computer Animation, July 2013, pp.125-133.

[78] Wilkie D, Sewall J, Lin M. Flow reconstruction for datadriven traffic simulation. ACM Trans. Graph, 2013, 32(4): Article No.89.

[79] Wolinski D, Guy S J, Olivier A H et al. Parameter estimation and comparative evaluation of crowd simulations. Computer Graphics Forum, 2014, 33(2): 303-312.

[80] Kapadia M, Singh S, Hewlett W et al. Egocentric affordance fields in pedestrian steering. In Proc. the 2009 Symposium on Interactive 3D Graphics and Games, Feb. 2009, pp.215-223.

[81] Yu Q, Terzopoulos D. A decision network framework for the behavioral animation of virtual humans. In Proc. the 2007 ACM SIGGRAPH/Eurographics Symposium on Computer Animation, 2007, pp.119-128.

[82] Kulpa R, Olivier A H, Ondrej J et al. Imperceptible relaxation of collision avoidance constraints in virtual crowds. ACM Trans. Graph., 2011, 30(6): Article No.138.

[83] Ondřej J, Pettre J, Olivier A H et al. A synthetic-vision based steering approach for crowd simulation. ACM Transactions on Graphics (TOG), 2010, 29(4): Article No.123.

[84] Metoyer R A, Hodgins J K. Reactive pedestrian path following from examples. The Visual Computer, 2004, 20(10): 635-649.

[85] Lee K H, Choi M G, Hong Q et al. Group behavior from video: A data-driven approach to crowd simulation. In Proc. the 2007 ACM SIGGRAPH/Eurographics Symposium on Computer Animation, Aug. 2007, pp.109-118. 
[86] Lerner A, Chrysanthou Y, Lischinski D. Crowds by example. Computer Graphics Forum, 2007, 26(3): 655-664.

[87] Li Y, Christie M, Siret O et al. Cloning crowd motions. In Proc. ACM SIGGRAPH/Eurographics Symposium on Computer Animation, July 2012, pp.201-210.

[88] Ju E, Choi M G, Park M et al. Morphable crowds. ACM Transactions on Graphics (TOG), 2010, 29(6): Article No.140.

[89] Flagg M, Rehg J M. Video-based crowd synthesis. IEEE Transactions on Visualization and Computer Graphics, 2013, 19(11): 1935-1947.

[90] Sun L, Li X, Qin W. Simulating realistic crowd based on agent trajectories. Computer Animation and Virtual Worlds, 2013, 24(3/4): 165-172.

[91] Kwon T, Lee K H, Lee J et al. Group motion editing. ACM Transactions on Graphics (TOG), 2008, 27(3): Article No.80.

[92] Lerner A, Fitusi E, Chrysanthou Y et al. Fitting behaviors to pedestrian simulations. In Proc. the 2009 ACM SIGGRAPH/Eurographics Symposium on Computer Animation, Aug. 2009, pp.199-208.

[93] Guy S J, van den Berg J, Liu W et al. A statistical similarity measure for aggregate crowd dynamics. ACM Transactions on Graphics (TOG), 2012, 31(6): Article No.190.

[94] Lai Y C, Chenney S, Fan S. Group motion graphs. In Proc. the 2005 ACM SIGGRAPH/Eurographics Symposium on Computer Animation, July 2005, pp.281-290.

[95] Courty N, Corpetti T. Crowd motion capture. Computer Animation and Virtual Worlds, 2007, 18(4-5): 361-370.

[96] Paris S, Pettré J, Donikian S. Pedestrian reactive navigation for crowd simulation: A predictive approach. Computer Graphics Forum, 2007, 26(3): 665-674.

[97] Chenney S. Flow tiles. In Proc. the 2004 ACM SIGGRAPH/Eurographics Symposium on Computer Animation, Aug. 2004, pp.233-242.

[98] Pelkey C D, Allbeck J M. Populating virtual semantic environments. Computer Animation and Virtual Worlds, 2014, 24(3/4): 405-412.

[99] Kraayenbrink N, Kessing J, Tutenel T et al. Semantic crowds. Entertainment Computing, 2014. (to be appeared)

[100] Golas A, Narain R, Lin M. Hybrid long-range collision avoidance for crowd simulation. In Proc. the 2013 ACM SIGGRAPH Symposium on Interactive $3 D$ Graphics and Games, March 2013, pp.29-36.

[101] Kim S, Guy S J, Lin M C et al. Predicting pedestrian trajectories using velocity-space reasoning. In Proc. the 10th Workshop on the Algorithm Foundations of Robotics, June 2012, pp. 609-623.

[102] Yeh H, Curtis S, Patil S et al. Composite agents. In Proc. the 2008 ACM SIGGRAPH/Eurographics Symposium on Computer Animation, July 2008, pp. 39-47.

[103] Lemercier S, Jelic A, Kupla R et al. Realistic following behaviors for crowd simulation. Computer Graphics Forum, 2012, 31(2): 489-498.

[104] Karamouzas I, Overmars M. Simulating and evaluating the local behavior of small pedestrian groups. IEEE Transactions on Visualization and Computer Graphics, 2012, 18(3): 394-406.

[105] Mao T, Jiang H, Xia S et al. Recent advances on human crowd simulation. International Journal of Virtual Reality, 2010, 9(3): 27-32.

[106] Jiang H, Xu W, Mao T et al. Continuum crowd simulation in complex environments. Computers \& Graphics, 2010, 34(5): 537-544.

[107] Jiang H, Xu W, Mao T et al. Crowds flow in complex environment. In Proc. the 11th IEEE International Conference on Computer-Aided Design and Computer Graphics, Aug. 2009, pp. 54-57.
[108] Jiang H, Xu W, Mao T et al. A semantic environment model for crowd simulation in multilayered complex environment. In Proc. the 16th ACM Symposium on Virtual Reality Software and Technology, Nov. 2009, pp.191-198.

[109] Mao T, Ye Q, Jiang $\mathrm{H}$ et al. Evoking panic in crowd simulation. Transactions on Edutainment VI, Pan Z, Cheok A D, Müller W (Eds.), 2011, pp.79-88.

[110] Jin X, Xu J, Wang C C L et al. Interactive control of largecrowd navigation in virtual environments using vector fields. IEEE Computer Graphics and Applications, 2008, 28(6): 3746.

[111] Jin X, Wang C C L, Huang S et al. Interactive control of real-time crowd navigation in virtual environment. In Proc. the 2007 ACM Symposium on Virtual Reality Software and Technology, Nov. 2007, pp.109-112.

[112] Gu Q, Deng Z. Context-aware motion diversification for crowd simulation. IEEE Computer Graphics and Applications, 2011, 31(5): 54-65.

[113] Gu Q, Deng Z. Generating freestyle group formations in agent-based crowd simulations. IEEE Computer Graphics and Applications, 2013, 33(1): 20-31.

[114] Zhang M, Xu M, Han L et al. Virtual Network Marathon with immersion, scientificalness, competitiveness, adaptability and learning. Computers \& Graphics, 2012, 36(3): 185-192.

[115] Xu J, Jin X, Yu Y et al. Shape-constrained flock animation. Computer Animation and Virtual Worlds, 2008, 19(3/4): 319-330.

[116] Xu M, Wu Y, Ye Y. Smooth and efficient crowd transformation. In Proc. the 20th ACM International Conference on Multimedia, Oct. 2012, pp.1189-1192.

[117] Xu M, Wu Y, Ye Y et al. Collective crowd formation transform with mutual information based runtime feedback. Computer Graphics Forum, 2014. (to be appeared)

[118] Aw A, Rascle M. Resurrection of "second order" models of traffic flow. SIAM Journal on Applied Mathematics, 2000, 60(3): 916-938.

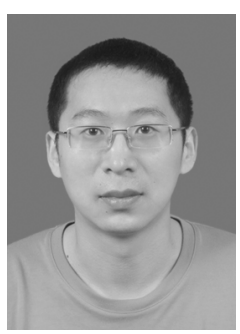

Ming-Liang $\mathbf{X u}$ is an associate professor in the School of Information Engineering of Zhengzhou University, China, and the secretary of the Virtual Reality Committee for the China Society of Image and Graphics. His research interests include computer animation, virtual and augment reality, and mobile computing. Xu got his Ph.D. degree in computer science and technology from the State Key Lab of CAD\&CG at Zhejiang University in 2012.

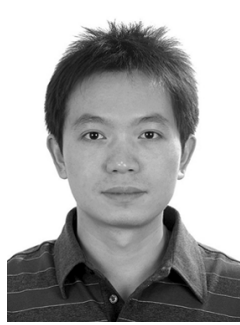

Hao Jiang is an assistant professor with Beijing Key Lab of Mobile Computing and Pervasive Devices, Institute of Computing Technology, Chinese Academy of Sciences, Beijing. He received his Ph.D. degree in computer science from Chinese Academy of Sciences in 2011. His research interests include crowd simulation, virtual reality, and intelligent human-computer interaction. He is a member of CCF, ACM and IEEE. 


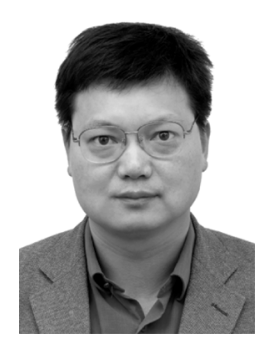

Xiao-Gang Jin received his B.S. degree in computer science, M.S. and Ph.D. degrees both in applied mathematics from Zhejiang University, Hangzhou, in 1989, 1992, and 1995, respectively. He is a professor with the State Key Lab of CAD\&CG, Zhejiang University. His current research interests include digital geometry processing, invisible orthodontics, crowd and group animation, cloth animation, video abstraction, implicit surface computing, special effects simulation, mesh fusion, and texture synthesis. He is a member of ACM and IEEE.

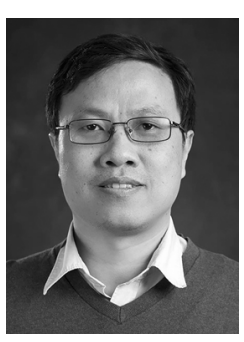

Zhigang Deng is currently an associate professor of computer science at the University of Houston, USA. His research interests include computer graphics, computer animation, virtual human modeling and animation, human computer interaction, and visual computing for biomedical applications. He received his Ph.D. degree in computer science at the University of Southern California in 2006, M.S. degree in computer science from Peking University in 2000, and B.S. degree in mathematics from Xiamen University in 1997. He is the recipient of a number of awards including Google Faculty Research Award, Texas Norman Hackerman Advanced Research Award, UH Teaching Excellence Award, NSFC Joint Research Fund for Overseas Chinese Scholars and Scholars in Hong Kong and Macao. His current research has been funded by the National Science Foundation of USA, National Institute of Health of USA, Texas NHARP Program, Google, Nokia, and other industry resources. Besides the CASA 2014 general co-chair and SCA 2015 general co-chair, currently he also serves as the associate editor of Computer Graphics Forum, and Computer Animation and Virtual Worlds Journal. He is a senior member of IEEE and a member of ACM. 